\title{
SOME NEW INEQUALITIES SIMILAR TO HARDY-HILBERT'S INEQUALITY
}

\author{
S. K. SUnANDA, C. NAHAK AND S. NANDA
}

Abstract. In this paper we have studied some new inequalities similar to Hardy-Hilbert's inequality. As applications, we have considered the associated integral inequalities.

Mathematics subject classification (2010): 26D15.

Keywords and phrases: Holder's inequality, Hardy-Hilbert's inequality, generalized $l_{p}$ space, generalized $L_{p}$ space.

\section{REFERENCES}

[1] YAng Bicheng, AND GaO Mingzhe, On a best value of Hardy-Hilbert's Inequality, Adv. Math. 26, No. 2(1997), 159-164.

[2] Yang Bicheng, And L. DeBnath, On new strengthened Hardy-Hilbert's Inequality, Int. J. Math. Math. Sci. 21, No. 1(1998), 403-408.

[3] YANG Bicheng, On an extension of Hardy-Hilbert's Inequality, Chin. Annal. Math. 23A (2002), 247-254.

[4] YAng Bicheng, On a New Inequality Similar to Hardy-Hilbert's Inequality, Math. Inequ. Appl. 6, No. 1(2003), 37-34.

[5] G. DAS, AND S. NANDA, Absolute almost convergence, Indian J. Maths, 34 (1992), 99-110.

[6] G. H. Hardy, J. E. LitTlewood And G. Polya, Inequalities, Cambridge University Press, Cambridge, MA, (1952).

[7] I. J. MADDOX, Elements of Functional Analysis, Cambridge University Press, (1970).

[8] Gao Mingzhe, And Yang Bicheng, On the extended Hilbert's Inequality, Proc. Amer. Math. Soc. 126, 3(1998), 751-759.

[9] S. Simons, The sequence spaces $l\left(p_{\gamma}\right)$ and $m\left(p_{v}\right)$, Proc. London Math. Soc. 13 (1965), 422-436.

[10] S. K. SunANDA, C. NAHAK AND S. NANDA, Some new generalizations of Hardy's integral inequality, Int. J. Math. and Math. Sci. Vol. 2006, Article ID 19013, 1-15. 